\title{
Coinfection pulmonary mucormycosis and aspergillosis with disseminated mucormycosis involving gastrointestinalin in an acute B-lymphoblastic leukemia patient
}

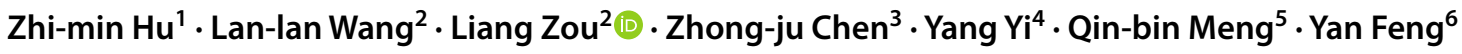

Received: 1 March 2021 / Accepted: 21 June 2021 / Published online: 3 July 2021

(c) Sociedade Brasileira de Microbiologia 2021

\begin{abstract}
Pulmonary mucormycosis and aspergillosis with disseminated mucormycosis involving gastrointestinalin is a very rare but lethal infection leading to extreme mortality. Herein, we present a unique case of pulmonary coinfection with Cunninghamella bertholletiae and Aspergillus flavus, with disseminated mucormycosis involving the jejunum caused by $C$. bertholletiae in an acute B-lymphocytic leukemia (B-ALL) patient with familial diabetes. Early administration of active antifungal agents at optimal doses and complete resection of all infected tissues led to improved therapeutic outcomes.
\end{abstract}

Keywords Mucormycosis, Aspergillosis · Disseminated mucormycosis $\cdot$ Acute lymphoblastic leukemia $\cdot$ Cunninghamella bertholletiae $\cdot$ Aspergillus flavus

Mucormycosis is a life-threatening and opportunistic infection leading to high mortality in immunocompromised individuals [1-3]. This lethal infection usually occurs in patients with uncontrolled diabetes, neutropenia, hematologic malignancies (HM), or corticosteroid treatment [4]. The incidence of mucormycosis has been increasing in recent decades,

Responsible Editor: Carlos Pelleschi Taborda

Liang Zou

zozozou@qq.com

1 Section of Clinical Microbiology and Molecular Biology, Department of Laboratory Medicine, Wuhan No. 1 Hospital, 215 Zhongshan Ave, Wuhan City 430022, Hubei, China

2 Section of Hematology, Department of Internal Medicine, Wuhan No. 1 Hospital, 215 Zhongshan Ave, Wuhan City 430022, Hubei, China

3 Section of Clinical Microbiology, Department of Laboratory Medicine, Tongji Hospital, Tongji Medicine College, Huazhong University of Science and Technology, 1095 Jiefang Ave, Wuhan, China

4 Department of Radiology, Wuhan No. 1 Hospital, 215 Zhongshan Ave, Wuhan City 430022, Hubei, China

5 Division of Gastrointestinal Surgery, Department of Surgery, Wuhan No. 1 Hospital, 215 Zhongshan Ave, Wuhan City 430022, Hubei, China

6 Department of Pathology, Wuhan No. 1 Hospital, 215 Zhongshan Ave, Wuhan City 430022, Hubei, China mainly due to the growth of the number of patients presenting with these predisposing conditions and medical advances in diagnosis $[1,5,6]$. In patients with $\mathrm{HM}$, the main clinical form is pulmonary mucormycosis (PM) [7-9]. The onset of $\mathrm{PM}$ is acute, the progression is rapid [10], and the reported mortality ranges from 20 to $100 \%$ in adults, depending on the underlying risk factors, site of infection, and treatment $[11,12]$. Gastrointestinal mucormycosis (GIM) is the least frequent form, constituting only $4-7 \%$ of all cases [13]. Because of the nonspecific clinical hallmarks of GIM, the diagnosis is often delayed or missed, and mortality remains high at 57\% [14]. However, in patients with prolonged neutropenia and in those with disseminated disease, mortality is $90-100 \%[4,15]$.

A 51-year-old female presented to the hematology clinic complaining of an approximately 1-month history of fatigue and reported a fever lasting for $24 \mathrm{~h}$. On admission, physical examination revealed a distended spleen. Other systemic examinations were unremarkable. At presentation, her body temperature was $37.4{ }^{\circ} \mathrm{C}$, her blood pressure was $115 / 71 \mathrm{mmHg}$, and her pulse was $80 \mathrm{bpm}$. Her blood work showed an elevated leukocyte count of $33.17 \times 10^{9} / \mathrm{L}$, hemoglobin $68 \mathrm{~g} / \mathrm{L}$, and platelets $44 \times 10^{9} / \mathrm{L}$, and the percentage of primitive cells was $95 \%$ in peripheral blood.

The timeline of diagnosis and targeted therapy is shown in Table 1. Fever was relieved by anti-biotherapy introduction. The common type of acute B-lymphocytic leukemia 
Table 1 Timeline of events

\begin{tabular}{|c|c|c|c|}
\hline Time & Clinical features & Biology results & Therapy strategies \\
\hline D1 & Fever & High level of CRP & $\begin{array}{l}\text { Levofloxacin } 0.6 \mathrm{~g} / \text { day and cefoperazone/sulbactam } \\
9 \text { g/day IV }\end{array}$ \\
\hline D3 & Bone marrow pathology & B-ALL & $\begin{array}{l}\text { Chemotherapy introduction (vindesine sulfate } 4 \mathrm{mg} \\
\text { per week and dexamethasone } 10 \mathrm{mg} / \text { day IV); } \\
\text { prophylactic therapy (fluconazole } 0.1 \mathrm{~g} / \text { day p.o.) }\end{array}$ \\
\hline D33 & Repeated bone marrow pathology & Not completely relieved & $\begin{array}{l}\text { Chemotherapy switched to IVCP (idarubicin } \\
10 \mathrm{mg} / \text { day } 1-3 \text {, vindesine sulfate } 4 \mathrm{mg} \text { per week, } \\
\text { CTX } 1.2 \mathrm{~g} / \text { day } 1,15 \text {, and dexamethasone } 10 \mathrm{mg} / \mathrm{c} \\
\text { IV) }\end{array}$ \\
\hline D39 & Neutropenia & & $\begin{array}{l}\text { Antifungal combined therapy (voriconazole } 0.4 \mathrm{~g} / \\
\text { day p.o.) }\end{array}$ \\
\hline D41 & Persistence of fever & High level of CRP & $\begin{array}{l}\text { Levofloxacin } 0.6 \mathrm{~g} / \text { day and cefoperazone/sulbactam } \\
9 \mathrm{~g} / \text { day IV }\end{array}$ \\
\hline D49 & Febrile neutropenia and cough & Rising of CRP rate; abnormal chest CT scan & $\begin{array}{l}\text { Switched anti-biotherapy therapy (meropenem } 3 \mathrm{~g} / \\
\text { day and linezolid } 1.2 \mathrm{~g} / \text { day IV) }\end{array}$ \\
\hline D51 & & $\begin{array}{l}\text { Filamentous fungi detected in BALF, serum } \\
\text { GM test positive }\end{array}$ & $\begin{array}{l}\text { Switched antifungal therapy (d-AmB } 0.4 \mathrm{mg} / \mathrm{kg} / \\
\text { day IV) }\end{array}$ \\
\hline D57 & Fever resolution & & \\
\hline D62 & Abdominal pain and fever & Blood pressure $85 / 33 \mathrm{mmHg}$; high level of CRP & $\begin{array}{l}\text { Adjusted to tigecycline } 0.1 \mathrm{~g} / \text { day, combined } \\
\mathrm{L}-\mathrm{AmB} 0.5 \mathrm{mg} / \mathrm{kg} / \text { day and voriconazole } 0.4 \mathrm{~g} / \\
\text { day IV }\end{array}$ \\
\hline D63 & & Abnormal abdominal CT scan, acute peritonitis & Emergency surgery \\
\hline D64 & & Hypha detected in jejunum histopathology & Adding L-AmB dose to $1.0 \mathrm{mg} / \mathrm{kg} /$ day IV \\
\hline D66 & Fever resolution & & \\
\hline D83 & Repeated bone marrow pathology & Completely relieved & Adding L-AmB dose to $1.2 \mathrm{mg} / \mathrm{kg} /$ day IV \\
\hline D100 & Regression of lesions on imagery & & $\begin{array}{l}\text { Switched antifungal therapy (posaconazole } 0.8 \mathrm{~g} / \\
\text { day p.o.) }\end{array}$ \\
\hline D130 & Complete remission & Negative BALF & \\
\hline
\end{tabular}

$D$ day, $C R P$ C-reactive protein, $I V$ intravenous, $B$ - $A L L$ acute B-lymphocytic leukemia, p.o. per os, $C T$ computed tomographic, $C T X$ cyclophosphamide, $d-A m B$ amphotericin B deoxycholate, $L-A m B$ liposomal amphotericin $B$, BALF bronchoalveolar lavage fluid, $G M$ galactomannan

(B-ALL) with the IKZF1 mutation was diagnosed by bone marrow pathology. Considering her history of familial diabetes and percutaneous coronary intervention, the chemotherapy program was initiated with a low dose of vindesine sulfate and dexamethasone and oral prophylactic treatment with fluconazole simultaneously. Serologies for (1,3)-betaD-glucan, galactomannan (GM) (Dynamiker Biotechnology Co., Ltd. Tianjin, China), syphilis, acquired immunodeficiency syndrome, and hepatitis A-E were negative. One month later, bone marrow pathology was repeated and showed $12 \%$ blast cells. A high-intensity IVCP program was performed. After 5 days, broad-spectrum antibiotics and voriconazole were started due to febrile neutropenia.

On day 49, significant pulmonary symptoms, such as productive cough, occurred, along with a persistent fever. Computed tomography (CT) showed a massive high-density shadow in the right superior lobe (Fig. 1) and rising levels of C-reactive protein (CRP). Blood culture was sterile, and polymerase chain reaction for cytomegalovirus and EB virus were negative. Anti-biotherapy was switched to meropenem and linezolid, but there was no obvious relief in

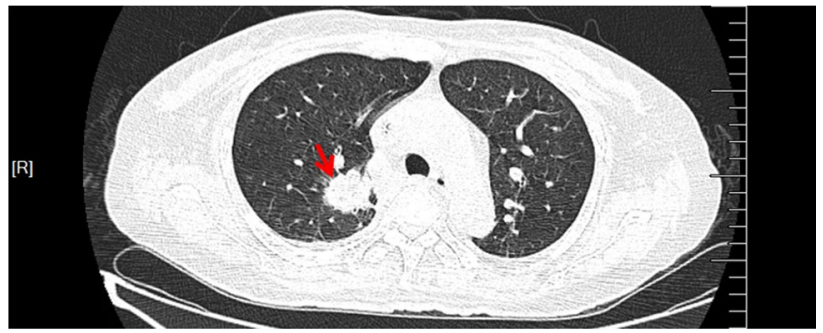

Fig. 1 A close-up chest CT scan of the right lung shows a massive high-density shadow (arrow) in the superior lobe

symptoms. On day 51, the serum GM antigen test was positive (2.38), and the microbiological tests were implemented with bronchoalveolar lavage fluid (BALF). Classically, microscopic evaluation with Gram (Fig. 2A) and calcofluor white (Fig. 2B) staining revealed filamentous hyphae; one type was uniformly thinner, septate, and branching at acute angles, and the other had a variable width, was nonseptate, and had branching filamentous hyphae and a ribbon-like appearance. Cultures of specimens on Sabouraud Dextrose 


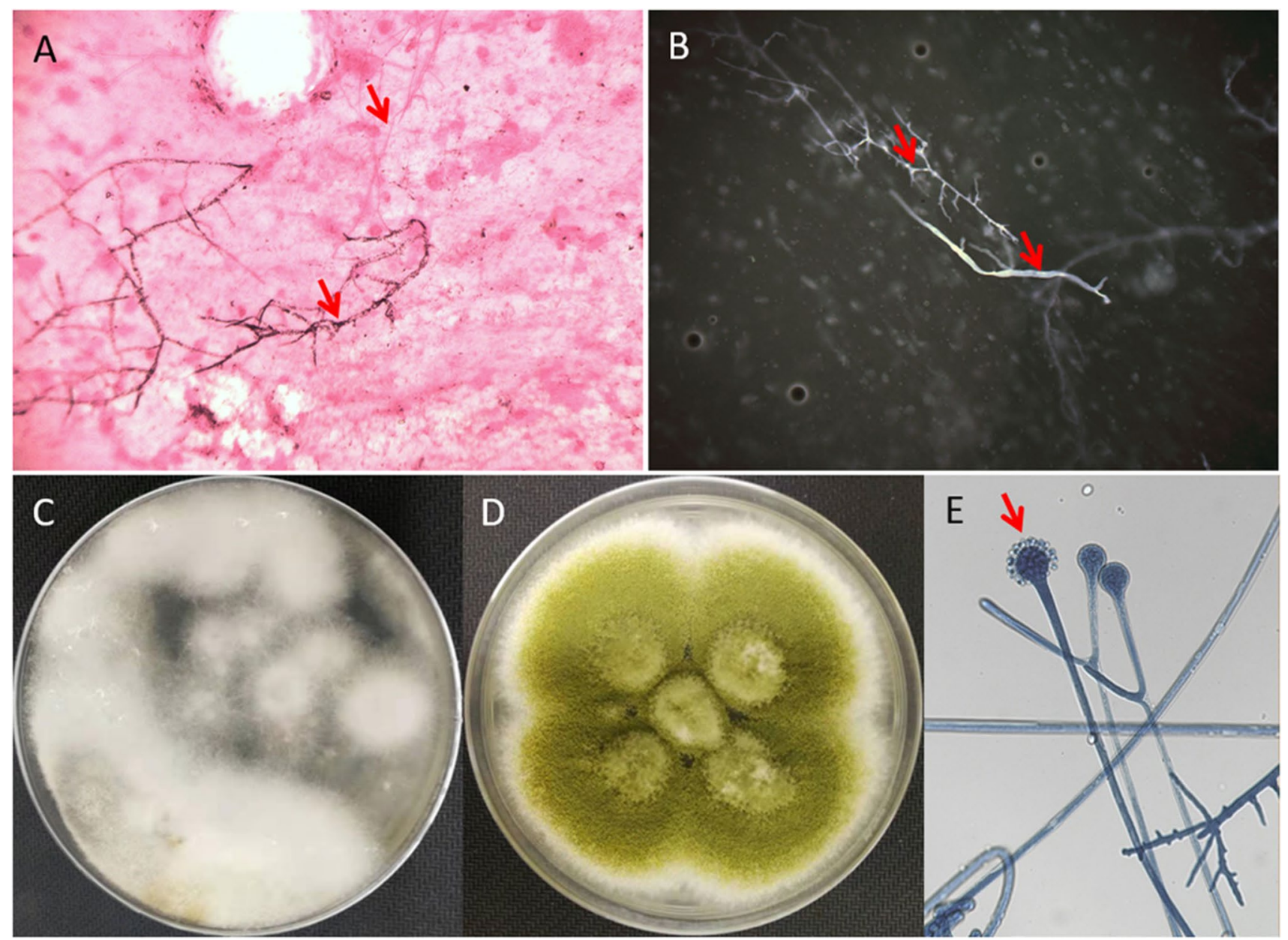

Fig. 2 (A) Gram staining and (B) calcofluor white staining showed two different hyphae: one is uniformly thinner, septate, and branching at acute angles. The other is a variable width with ribbon-like appearance $(400 \times$ magnification). (C) Cunninghamella bertholletiae and

Agar (SDA) showed the features as Mucorales. Colonies appeared cotton and white-gray, both on the surface and reverse side (Fig. 2C). Lactophenol cotton blue staining revealed irregularly branching sporangiophores terminating prominently, and sporangioles borne off the vesicles (Fig. 2E). Cunninghamella bertholletiae was identified by mycological characteristics and internal transcribed spacer (ITS)-based sequencing (accession no.MT470208). DNA sequences were analyzed using NCBI BLAST (https://blast. ncbi.nlm.nih.gov/Blast.cgi). Another pathogen isolated from BALF was Aspergillus flavus (accession no. MW911813).

Based on the characteristics of the filamentous hyphae, we switched the antifungal therapy to intravenous amphotericin $\mathrm{B}$ deoxycholate $(\mathrm{d}-\mathrm{AmB})$ with an initial dose of $0.5 \mathrm{mg} / \mathrm{kg} /$ day. Persistent fever was resolved, but unexpectedly, acute abdominal pain with high fever and a "sudden drop" in blood pressure appeared on day 62. Anti-biotherapy was adjusted to tigecycline combined with liposomal
(D) A. flavus isolated from BALF cultured on a SDA medium plate for $48 \mathrm{~h}$ at $35{ }^{\circ} \mathrm{C}$. (E) Cunninghamella bertholletiae sporangiophores in terminal swellings called vesicles, with sporangioles (lactophenol cotton blue staining, $400 \times$ magnification)

amphotericin B (L-AmB). At midnight, the abdominal pain worsened, and acute diffuse peritonitis was considered. CT showed some free abdominal gas under the diaphragm, and peritoneal fluid was detected (Fig. 3). Emergency surgical management, including partial resection of the jejunum and ileum, was performed. There were 9 perforations in the jejunum 190-210 cm from the curved ligament, with an aperture of approximately $1-2 \mathrm{~cm}$, and a perforated ileum was detected approximately $25 \mathrm{~cm}$ from the ileocecal part.

Histopathology of specimens from the jejunum and ileum showed broad septate fungal hyphae (Fig. 4). Cultures of specimens from the jejunum also showed features such as Mucorales, and C. bertholletiae (accession no. MW915438) was identified according to the same protocols mentioned above. Antifungal susceptibility tests according to the Clinical and Laboratory Standards Institute (CLSI) M38-A2 broth microdilution document [16] were implemented. The susceptibility profiles of $C$. bertholletiae isolated from BALF 


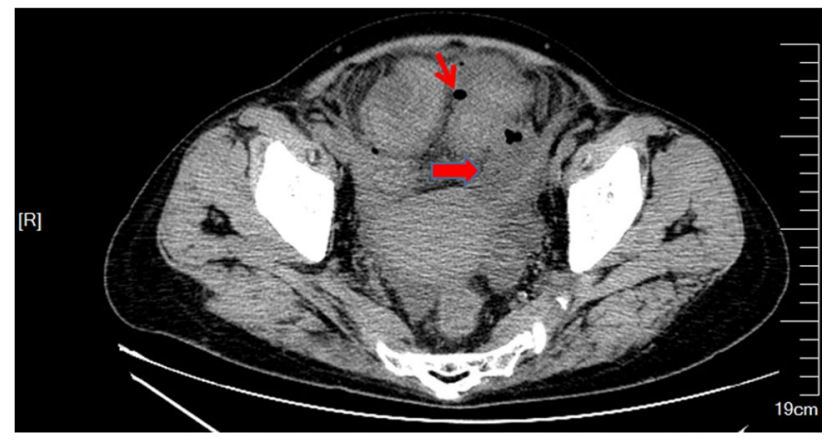

Fig. 3 The thinner arrow shows free abdominal gas under the diaphragm, and the wider arrow shows peritoneal fluid

and tissue showed fluconazole $256 \mu \mathrm{g} / \mathrm{ml}$, itraconazole $0.5 \mu \mathrm{g} / \mathrm{ml}$, posaconazole $0.5 \mu \mathrm{g} / \mathrm{ml}$, voriconazole $8 \mu \mathrm{g} / \mathrm{ml}$, AmB $2 \mu \mathrm{g} / \mathrm{ml}$, and flucytosine $64 \mu \mathrm{g} / \mathrm{ml}$. The susceptibility profiles of A. flavus isolated from BALF showed itraconazole $1 \mu \mathrm{g} / \mathrm{ml}$, posaconazole $0.5 \mu \mathrm{g} / \mathrm{ml}$, voriconazole $0.25 \mu \mathrm{g} /$ $\mathrm{ml}$, and $\mathrm{AmB} 2 \mu \mathrm{g} / \mathrm{ml}$.

$\mathrm{L}-\mathrm{AmB}$ was added to $1.0 \mathrm{mg} / \mathrm{kg} / \mathrm{day}$ for 1 week, followed by fever resolution. She was covered pre- and postsurgery with L-AmB for 8 weeks. Considering the relief of symptoms and regression of lesions on imagery, our strategy switched to oral posaconazole $0.8 \mathrm{~g} /$ day. The patient was discharged in good condition for continuous therapy with oral posaconazole $0.8 \mathrm{~g} /$ day for almost 6 months. Due to the COVID-19 pandemic and other reasons, the patient's family finally gave up and the patient passed away at home last year. Mucormycosis and aspergillosis are opportunistic fungal infections that can lead to life-threatening complications and occur most commonly in individuals with neutropenia and prolonged immunosuppressive therapy [17]. An epidemiological article of 929 cases of mucormycosis found a correlation between the patient survival and the species within
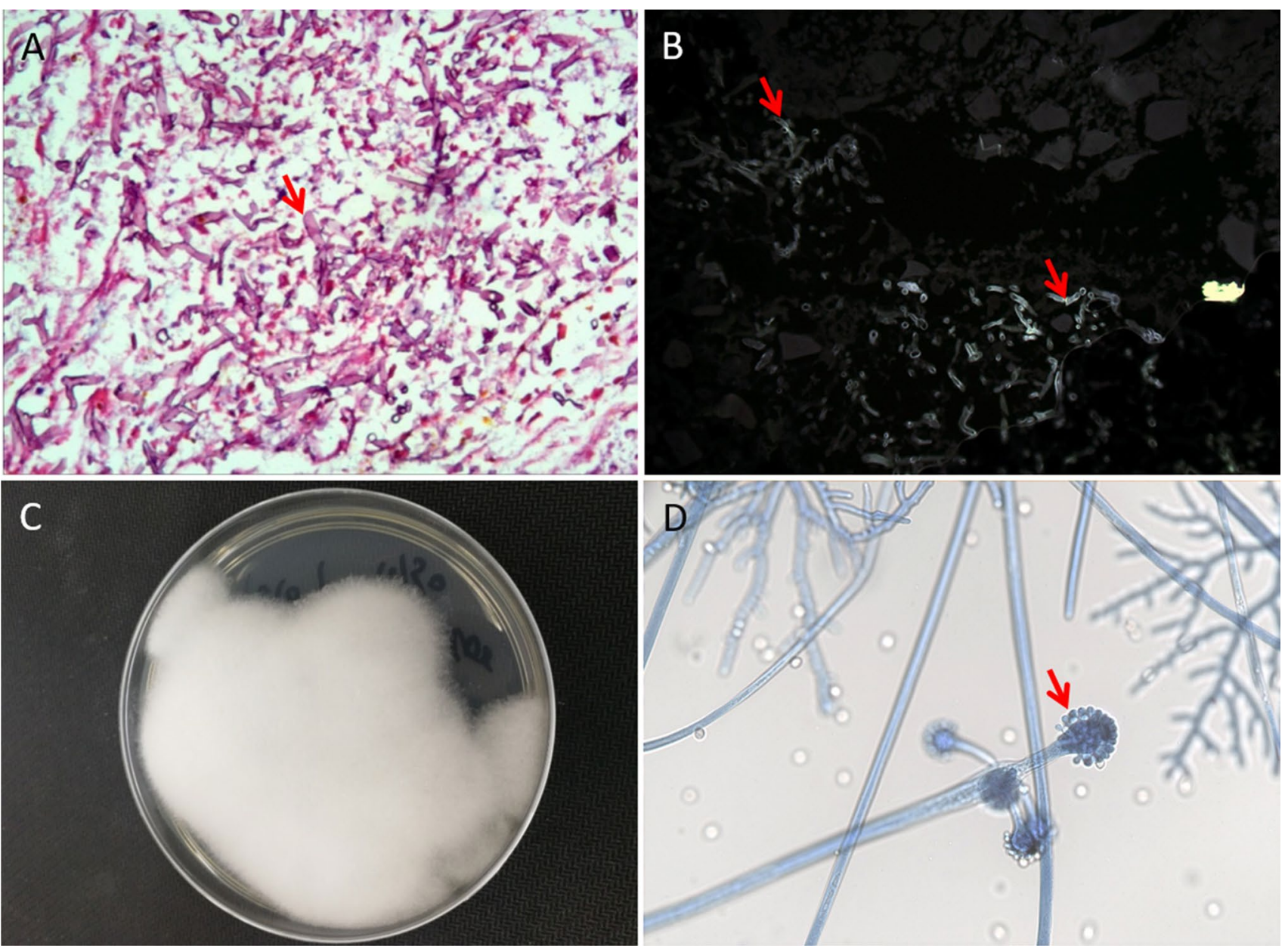

Fig. 4 Photomicrographs from the jejunum showed acute necrotizing angioinvasion with abundant broad, nonseptate fungal hyphae (arrow) consistent with mucormycosis ((A) hematoxylin and eosin staining; (B) calcofluor white staining; $400 \times$ magnification). (C) Cunningha- mella bertholletiae colony isolated from tissue cultured on a SDA medium plate for $48 \mathrm{~h}$ at $35^{\circ} \mathrm{C}$. (D) Lactophenol cotton blue staining revealed irregularly branching sporangiophores terminating prominently, and sporangioles borne off the vesicles $(400 \times$ magnification $)$ 
the Mucorales, given the conclusion of Cunninghamella spp. causing the highest percentage of crude mortalities and being an independent risk factor for death in the multivariate analysis [18]. As the most representative etiologic agent, C. bertholletiae occurs less frequently but causes refractory and fatal infections. A review of 15 cases of mucormycosis caused by Cunninghamella spp. indicated a patient population mainly consisting of neutropenia and transplantation [19]. GIM is the rarest type of Mucorales infection, and the successful management of the aggressive illness requires early surgical debridement, control of underlying disease, and suitable antifungal therapy [20]. A typical characteristic of pathophysiology of Mucorales infection is angioinvasion with thrombosis and thus necrosis of an affected part of the intestine. This will produce acute abdominal pain, possible bleeding, or perforation [20, 21]. To the best of our knowledge, this is the first report of pulmonary coinfection with $C$. bertholletiae and A. flavus with disseminated mucormycosis involving the jejunum caused by $C$. bertholletiae in a B-ALL patient in China.

Cunninghamella bertholletiae demonstrated to be the most resistant species among zygomycetes. Literature on C. bertholletiae indicates higher minimum inhibitory concentrations (MICs); $37 \%$ of the isolates had MICs of $2 \mu \mathrm{g} / \mathrm{ml}$ for $\mathrm{AmB}$, while approximately $75 \%$ of the isolates appeared to be susceptible to posaconazole [22]. The high MICs to AMB and the low MICs for itraconazole and posaconazole against $C$. bertholletiae have been reported [22-25]. In general, our results agree with other studies [22-25]. Although decreased susceptibility to amphotericin B in vitro, the lipid formulations of AMB may achieve higher concentrations in vivo [22]. According to the epidemiologic cutoff values (ECVs) of antifungals for A. flavus [26], the strain isolated from BALF could be considered a wild-type. Early diagnosis of mucormycosis is the key to treatment and prognosis. And the definitive diagnosis of mucormycosis depends on a combination of histopathological findings and standard mycological methods, as well as DNA sequencing of the ITS region, which has been suggested as a valuable target for identification at the genus and the species level by the CLSI guidelines [27]. Successful management of mucormycosis is on the basis of a multimodal manner, including reversal or revocation of underlying predisposing factors, early administration of suitable antifungal agents, and thorough resection of all infected tissues $[28,29]$. According to the guidelines of European Conference on Infections in Leukaemia (ECIL6) and European Confederation of Medical Mycology-The European Society for Clinical Microbiology and Infectious Diseases (ECMM-ESCMID), d-AmB and L-AmB are recommended as the first-line antifungal agent approved for the therapy of invasive mucormycosis [30]. High-dose L-AmB $(10 \mathrm{mg} / \mathrm{kg} /$ day) immediately administered upon suspicion of mucormycosis greatly suppressed the infection in its early stage [31]. However, in the absence of surgical debridement for infected tissue, antifungal therapy alone is rarely curative [4].

Our aim in this report is to highlight the need for a high clinical suspicion for Mucorales infection in neutropenic, immunocompromised, and diabetic patients. Direct microscopic testing with calcofluor white is the key to rapid diagnosis. In addition, effective multidepartmental communication with consulting physicians, such as hematologists, pulmonologists, and microbiologists, as well as immediate initiation of treatment, including surgical resection, can lead to improved patient outcomes in managing this rare but devastating disease and lay a solid foundation for the subsequent treatment of original disease.

Funding This work was supported by the Wuhan Health Scientific Research Key Project (Grant No. WX18A06).

\section{Declarations}

Conflict of interest The authors declare no competing interests.

\section{References}

1. Bitar D, Lortholary O, Le Strat Y, Nicolau J, Coignard B, Tattevin $P$ et al (2014) Population based analysis of invasive fungal infections, france, 2001-2010. Emerg Infect Dis 20:1149-1155

2. Pana ZD, Seidel D, Skiada A, Groll AH, Petrikkos G, Cornely OA et al (2016) Invasive mucormycosis in children: an epidemiologic study in European and non-European countries based on two registries. BMC Infect Dis 16:667

3. Ibrahim AS, Voelz K (2017) The mucormycete-host interface. Curr Opin Microbiol 40:40-45

4. Ibrahim AS (2014) Host-iron assimilation: pathogenesis and novel therapies of mucormycosis. Mycoses 57(Suppl 3):13-17

5. Petrikkos G, Skiada A, Lortholary O, Roilides E, Walsh TJ, Kontoyiannis DP (2012) Epidemiology and clinical manifestations of mucormycosis. Clin Infect Dis 54(Suppl 1):S23-34

6. Warkentien T, Rodriguez C, Lloyd B, Wells J, Weintrob A, Dunne JR et al (2012) Invasive mold infections following combat-related injuries. Clin Infect Dis 55:1441-1449

7. Skiada A, Pagano L, Groll A, Zimmerli S, Dupont B, Lagrou K et al (2011) Zygomycosis in Europe: analysis of 230 cases accrued by the registry of the European Confederation of Medical Mycology (ECMM) Working Group on Zygomycosis between 2005 and 2007. Clin Microbiol Infect 17:1859-1867

8. Lanternier F, Dannaoui E, Morizot G, Elie C, Garcia-Hermoso $D$, Huerre $M$ et al (2012) A global analysis of mucormycosis in France: the RetroZygo study (2005-2007). Clin Infect Dis 54(Suppl 1):S35-43

9. Klimko NN, Khostelidi SN, Volkova AG, Popova MO, Bogomolova TS, Zuborovskaya LS et al (2014) Mucormycosis in haematological patients: case report and results of prospective study in Saint Petersburg, Russia. Mycoses 57(Suppl 3):91-96

10. Li YH, Sun P, Guo JC (2017) Clinical analysis of diabetic combined pulmonary mucormycosis. Mycopathologia 182:1111-1117 
11. Hammond SP, Baden LR, Marty FM (2011) Mortality in hematologic malignancy and hematopoietic stem cell transplant patients with mucormycosis, 2001 to 2009. Antimicrob Agents Chemother 55:5018-5021

12. Zilberberg MD, Shorr AF, Huang H, Chaudhari P, Paly VF, Menzin J (2014) Hospital days, hospitalization costs, and inpatient mortality among patients with mucormycosis: a retrospective analysis of US hospital discharge data. BMC Infect Dis 14:310-319

13. Martinello M, Nelson A, Bignold L, Shaw D (2012) "We are what we eat!' Invasive intestinal mucormycosis: a case report and review of literature. Med Mycol Case Rep 1:52-55

14. Dioverti MV, Cawcutt KA, Abidi M, Sohail MR, Walker RC, Osmon DR (2015) Gastrointestinal mucormycosis in immunocompromised hosts. Mycoses 58:714-718

15. Ibrahim AS, Spellberg B, Walsh TJ, Kontoyiannis DP (2012) Pathogenesis of mucormycosis. Clin Infect Dis 54(Suppl 1):S16-S22

16. Clinical and Laboratory Standards Institute (2008) Reference method for broth dilution antifungal susceptibility testing of filamentous fungi. 2nd ed. CLSI document M38-A2. Clinical and Laboratory Standards Institute, Wayne

17. Chermetz M, Gobbo M, Rupel K, Ottaviani G, Tirelli G, Bussani $\mathrm{R}$ et al (2016) Combined orofacial aspergillosis and mucormycosis: fatal complication of a recurrent paediatric glioma-case report and review of literature. Mycopathologia 181:723-733

18. Roden MM, Zaoutis TE, Buchanan WL, Knudsen TA, Sarkisova TA, Schaufele RL et al (2005) Epidemiology and outcome of zygomycosis: a review of 929 reported cases. Clin Infect Dis 41:634-653

19. Petraitis V, Petraitiene R, Antachopoulos C, Hughes JE, Cotton MP, Kasai M et al (2013) Increased virulence of Cunninghamella bertholletiae in experimental pulmonary mucormycosis: correlation with circulating molecular biomarkers, sporangiospore germination and hyphal metabolism. Med Mycol 51:72-82

20. Goel P, Jain V, Sengar M, Mohta A, Das P, Bansal P (2013) Gastrointestinal mucormycosis: a success story and appraisal of concepts. J Infect Public Health 6:58-61

21. Sun M, Hou X, Wang X, Chen G, Zhao Y (2017) Gastrointestinal mucormycosis of the jejunum in an immunocompetent patient. Medicine (Baltimore) 96:e6360

22. Almyroudis NG, Sutton DA, Fothergill AW, Rinaldi MG, Kusne S (2007) In vitro susceptibilities of 217 clinical isolates of zygomycetes to conventional and new antifungal agents. Antimicrob Agents Chemother 51:2587-2590
23. Alastruey-Izquierdo A, Castelli MV, Cuesta I, Monzon A, CuencaEstrella M, Rodriguez-Tudela JL (2009) Activity of posaconazole and other antifungal agents against Mucorales strains identified by sequencing of internal transcribed spacers. Antimicrob Agents Chemother 53:1686-1689

24. Pastor FJ, Ruíz-Cendoya M, Pujol I, Mayayo E, Sutton DA, Guarro J (2010) In vitro and In vivo antifungal susceptibilities of the Mucoralean fungus Cunninghamella. Antimicrob Agents Chemother 54:4550-4555

25. Espinel-Ingroff A, Chakrabarti A, Chowdhary A, Cordoba S, Dannaoui E, Dufresne P et al (2015) Multicenter evaluation of MIC distributions for epidemiologic cutoff value definition to detect amphotericin B, posaconazole, and itraconazole resistance among the most clinically relevant species of Mucorales. Antimicrob Agents Chemother 59:1745-1750

26. Clinical and Laboratory Standards Institute (2020) Performance standards for antifungal susceptibility testing of filamentous fungi. M61, 2nd edn. Clinical and Laboratory Standards Institute, Wayne

27. Dannaoui E (2009) Molecular tools for identification of zygomycetes and the diagnosis of zygomycosis. Clin Microbiol Infect 15(Suppl 5):66-70

28. Cornely OA, Arikan-Akdagli S, Dannaoui E, Groll AH, Lagrou K, Chakrabarti A et al (2014) ESCMID and ECMM joint clinical guidelines for the diagnosis and management of mucormycosis 2013. Clin Microbiol Infect 20(Suppl 3):5-26

29. Tissot F, Agrawal S, Pagano L, Petrikkos G, Groll AH, Skiada A et al (2017) ECIL-6 guidelines for the treatment of invasive candidiasis, aspergillosis and mucormycosis in leukemia and hematopoietic stem cell transplant patients. Haematologica 102:433-444

30. Skiada A, Lass-Floerl C, Klimko N, Ibrahim A, Roilides E, Petrikkos G (2018) Challenges in the diagnosis and treatment of mucormycosis. Med Mycol 56:S93-S101

31. Ota H, Yamamoto H, Kimura M, Araoka H, Fujii T, Umeyama T et al (2017) Successful treatment of pulmonary mucormycosis caused by Cunninghamella bertholletiae with high-dose liposomal amphotericin B (10 mg/kg/day) followed by a lobectomy in cord blood transplant recipients. Mycopathologia 182:847-853

Publisher's note Springer Nature remains neutral with regard to jurisdictional claims in published maps and institutional affiliations. 